\title{
Analysis of Oxoglaucine in the Treatment of Breast Cancer Based on Network Pharmacology and Bioinformatics
}

\author{
Ting Chen ${ }^{2}$, Haiyu Chen ${ }^{1}$, Liang Zhang ${ }^{1}$, Bin Zhou ${ }^{2}$, Chao Yang ${ }^{2}$, Xulong Huang ${ }^{1}$, Bin Huang ${ }^{1, *}$ \\ ${ }^{1}$ School of Pharmaceutical Sciences, Hunan University of Medicine, Huaihua 418000, China \\ ${ }^{2}$ Department of Pharmacy, Guizhou Health Vocational College, Tongren 554301, China
}

\begin{abstract}
To explore the potential molecular mechanism of Oxoglaucine(OG) in the treatment of Breast Cancer(BC) based on network pharmacology and bioinformatics. TCMSP and SwissTargetPrediction databases search for OG Related targets, and GeneCards database finds all BC-related targets. Take the intersection of $\mathrm{OG}$ and $\mathrm{BC}$ as all potential targets that inhibit $\mathrm{BC}$. All potential targets are topologically analyzed by Cytoscape 3.7.1 software, and finally the core target is obtained. The start analysisi function in the DAVID database performs bioinformatics analysis on all core targets, and further visualizes them with the help of $\mathrm{R}$ language tools. As a result, 104 potential targets were obtained, of which SRC, PIK3CA, EGFR, MTOR, ESR1, MAPK1, PTGS2, AR, and NOS3 were the main core targets. OG inhibits the occurrence of BC through Pathways in cancer, PI3K-Akt signaling pathway, Proteoglycans in cancer, ErbB signaling pathway, HIF-1 signaling pathway related pathways, mainly involving signal transduction, protein phosphorylation, negative regulation of apoptotic process, positive regulation of transcription from RNA polymerase II promoter, phosphatidylinositol-mediated signaling biological processes. This study initially reveals the molecular mechanism of $\mathrm{OG}$ inhibiting $\mathrm{BC}$, which provides a reference for further research.
\end{abstract}

\section{Introduction}

Breast Cancer(BC) is a common and multiple malignant tumor all over the world, which seriously endangers the life and health of women[1]. BC originates from the malignant transformation of breast ductal epithelium or breast acinar epithelial cells, distant metastasis is one of the main causes of death[2-3]. The conventional treatment of BC is mainly based on radiotherapy and chemotherapy. Some patients will also cooperate with other drugs to improve their immune function after surgery[4-5]. The 2019 BC Diagnosis and Treatment Guidelines propose that preoperative neoadjuvant drugs (anthracyclines, taxanes) treatment can be selected according to the situation[6], so the development of new natural medicines is of great significance for the adjuvant treatment of $\mathrm{BC}$.

Oxoglaucine $(\mathrm{OG})$ is an alkaloid component isolated from the traditional Chinese medicine Corydalis yanhusuo[7]. OG has strong pharmacological activity. Modern research shows that OG has antiviral, antibacterial, anti-inflammatory, immune repair and other activities[8-11], but its anti-tumor effects are rarely reported, and the mechanism of action is still unclear.

This study mainly used the methods of network pharmacology and bioinformatics to predict the molecular mechanism of $\mathrm{OG}$ inhibiting $\mathrm{BC}$, and provide reference for the in-depth study of $\mathrm{OG}$ anti-tumor.

\section{Materials and methods}

\subsection{OG-related targets}

We obtain all the active targets of OG from TCMSP and SwissTargetPrediction(http://www.swisstargetprediction. $\mathrm{ch} /$ ) database, and establish a data set.

\subsection{BC-related targets}

We obtained BC-related targets in the GeneCards database (https://www.genecards.org/), searched for the keyword "breast cancer", and established a disease target data set.

\subsection{Network construction}

Potential targets for inhibiting BC are obtained by taking the intersection of disease and drug target data sets. The String database (https://string-db.org/) is used to construct the PPI network of potential targets, PPI network is analyzed using cytoscape 3.7.1 software to calculate the "Degree", "Betweenness centrality" and "Closeness centrality", Obtain all core targets, and use greater than the median as the screening condition.

\footnotetext{
* Corresponding author: huangbinsg@163.com
} 


\subsection{Bioinformatic analysis}

The core targets are entered in DAVID (https://david. ncifcrf.gov/tools.jsp)for Genetic ontology (GO) enrichment analysis and Kyoto Encyclopedia of Genes and Genomes (KEGG) pathway analysis. Using R language tools to visually analyze the core targets, $\mathrm{P} \leq$ 0.05 as the screening criterion.

\subsection{Pathway mapper construction}

KEGG (https://www.genome.jp/kegg/) Mapper function will map core targets to different pathways.

\section{Results}

\subsection{Target prediction}

In the TCMSP and Swiss Target Prediction databases, 112 targets corresponding to OG were obtained, and 15207 BC targets were obtained in the GeneCards database. The disease and drug data sets were intersected to obtain 104 potential targets. The results are shown in Figure 1.

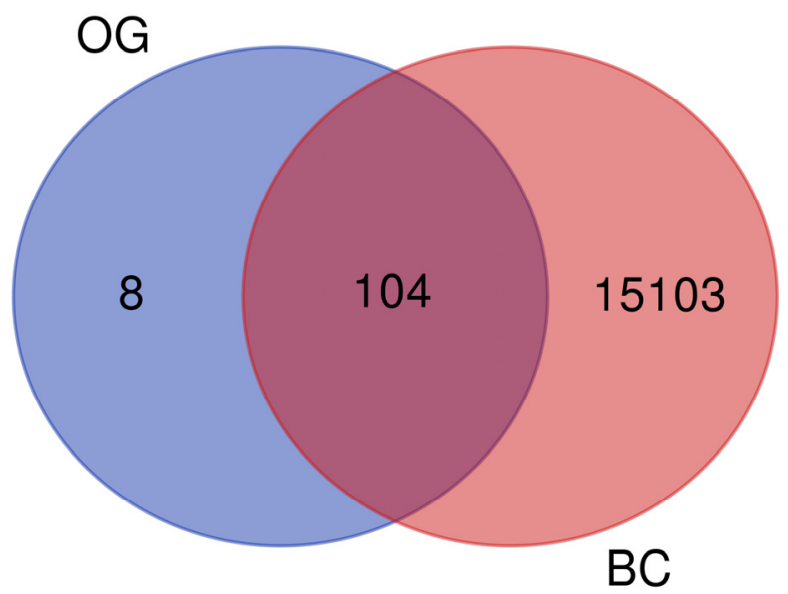

Fig. 1. Drug-disease intersection target Venn diagram

\subsection{Screening of core targets}

We use Cytoscape 3.7.1 software to visually analyze potential targets, the core target must be greater than the median of the three topological parameters at the same time. The median values of the three topological parameters are 10 (Degree), 0.0029 (Betweenness centrality), 0.4525 (Closeness centrality). The results are shown in Table 1 and Figure 3.

\subsection{Drug-disease-core target network constru- ction}

We use Cytoscape 3.7.1 software to build a "drugdisease-core target" network, and the results are shown in Figure 2.

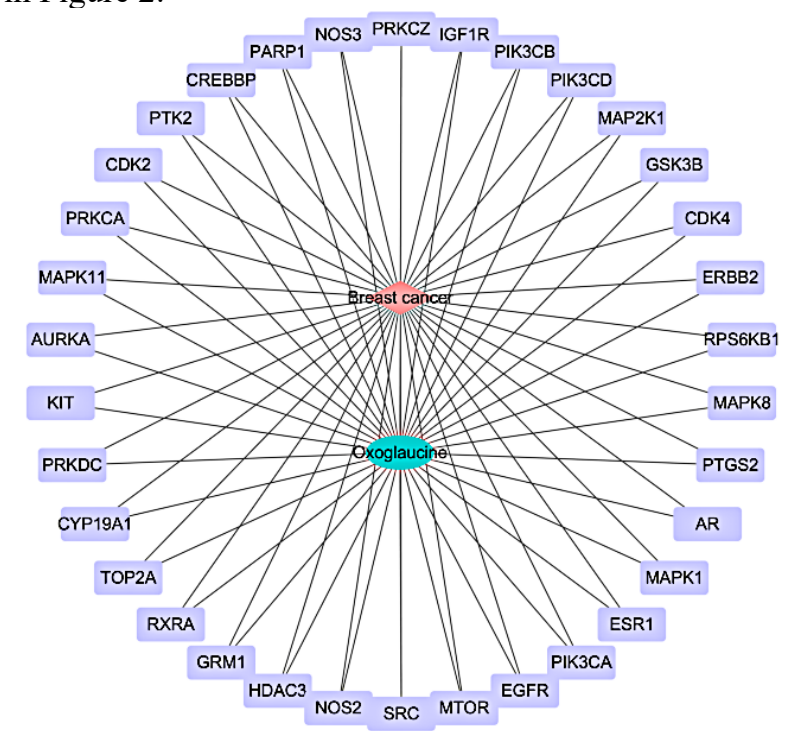

Fig.2. Drugs-diseases-core targets network

\subsection{GO Biological process enrichment analysis}

Enrichment analysis was performed on 171 biological processes, and the top 10 biological processes, cell composition and molecular functions were selected. The results are shown in Figure 4. The results preliminary reveals that $\mathrm{OG}$ may inhibit $\mathrm{BC}$ through a variety of biological processes such as signal transduction, protein phosphorylation, negative regulation of apoptotic process, positive regulation of transcription from RNA polymerase II promoter, phosphatidylinositol-mediated signaling.

Table 1. Related topological parameters of the core target

\begin{tabular}{|c|c|c|c|c|c|}
\hline $\begin{array}{l}\text { UniProt } \\
\text { CID }\end{array}$ & $\begin{array}{l}\text { Gene } \\
\text { name }\end{array}$ & Protein name & $\begin{array}{l}\text { Degr } \\
\text { ee }\end{array}$ & $\begin{array}{l}\text { Closeness } \\
\text { Centrality }\end{array}$ & $\begin{array}{l}\text { Betweenness } \\
\text { Centrality }\end{array}$ \\
\hline P12931 & SRC & $\begin{array}{l}\text { SRC Proto-Oncogene, Non-Receptor } \\
\text { Tyrosine Kinase }\end{array}$ & 50 & 0.6536 & 0.1270 \\
\hline P11511 & PIK3CA & $\begin{array}{l}\text { Phosphatidylinositol-4, 5-Bisphosphate } \\
\text { 3-Kinase Catalytic Subunit Alpha }\end{array}$ & 42 & 0.6173 & 0.0976 \\
\hline P00533 & EGFR & Epidermal Growth Factor Receptor & 42 & 0.6173 & 0.0800 \\
\hline P42345 & MTOR & Mechanistic Target Of Rapamycin Kinase & 46 & 0.6329 & 0.0783 \\
\hline P03372 & ESR1 & Estrogen Receptor 1 & 39 & 0.5988 & 0.0701 \\
\hline P28482 & MAPK1 & Mitogen-Activated Protein Kinase 1 & 39 & 0.5988 & 0.0674 \\
\hline P35354 & PTGS2 & Prostaglandin-Endoperoxide Synthase 2 & 30 & 0.5618 & 0.0611 \\
\hline P10275 & AR & Androgen Receptor & 33 & 0.5714 & 0.0540 \\
\hline P29474 & NOS3 & Nitric Oxide Synthase 3 & 24 & 0.5376 & 0.0536 \\
\hline P09874 & PARP1 & Poly(ADP-Ribose) Polymerase 1 & 23 & 0.5236 & 0.0392 \\
\hline
\end{tabular}




\begin{tabular}{|c|c|c|c|c|c|}
\hline P11511 & CYP19A1 & $\begin{array}{l}\text { Cytochrome P450 Family } 19 \text { Subfamily A } \\
\text { Member } 1\end{array}$ & 15 & 0.5051 & 0.0315 \\
\hline P49841 & GSK3B & Glycogen Synthase Kinase 3 Beta & 26 & 0.5405 & 0.0276 \\
\hline P11802 & CDK4 & Cyclin Dependent Kinase 4 & 26 & 0.5291 & 0.0259 \\
\hline P17252 & PRKCA & Protein Kinase C Alpha & 21 & 0.5208 & 0.0252 \\
\hline Q13255 & GRM1 & Glutamate Metabotropic Receptor 1 & 12 & 0.4566 & 0.0236 \\
\hline Q92793 & CREBBP & CREB Binding Protein & 22 & 0.5208 & 0.0208 \\
\hline P45983 & MAPK8 & Mitogen-Activated Protein Kinase 8 & 30 & 0.5495 & 0.0198 \\
\hline O00329 & PIK3CD & $\begin{array}{l}\text { Phosphatidylinositol-4,5-Bisphosphate } \\
\text { 3-Kinase Catalytic Subunit Delta }\end{array}$ & 26 & 0.5051 & 0.0191 \\
\hline P24941 & CDK2 & Cyclin Dependent Kinase 2 & 21 & 0.5051 & 0.0144 \\
\hline Q05513 & PRKCZ & Protein Kinase C Zeta & 25 & 0.5376 & 0.0133 \\
\hline P19793 & RXRA & Retinoid X Receptor Alpha & 13 & 0.4717 & 0.0128 \\
\hline P42338 & PIK3CB & $\begin{array}{l}\text { Phosphatidylinositol-4,5-Bisphosphate } \\
\text { 3-Kinase Catalytic Subunit Beta }\end{array}$ & 26 & 0.5102 & 0.0107 \\
\hline P23443 & RPS6KB1 & Ribosomal Protein S6 Kinase B1 & 27 & 0.5525 & 0.0102 \\
\hline P04626 & ERBB2 & Erb-B2 Receptor Tyrosine Kinase 2 & 26 & 0.5405 & 0.0095 \\
\hline P78527 & PRKDC & $\begin{array}{l}\text { Protein Kinase, DNA-Activated, Catalytic } \\
\text { Subunit }\end{array}$ & 15 & 0.4831 & 0.0092 \\
\hline O14965 & AURKA & Aurora Kinase A & 17 & 0.4926 & 0.0088 \\
\hline Q02750 & MAP2K1 & Mitogen-Activated Protein Kinase Kinase 1 & 26 & 0.5376 & 0.0086 \\
\hline Q05397 & PTK2 & Protein Tyrosine Kinase 2 & 22 & 0.5181 & 0.0073 \\
\hline O15379 & HDAC3 & Histone Deacetylase 3 & 12 & 0.4808 & 0.0071 \\
\hline P11388 & TOP2A & DNA Topoisomerase II Alpha & 15 & 0.4926 & 0.0057 \\
\hline Q15759 & MAPK11 & Mitogen-Activated Protein Kinase 11 & 18 & 0.4926 & 0.0053 \\
\hline P08069 & IGF1R & Insulin Like Growth Factor 1 Receptor & 25 & 0.5376 & 0.0050 \\
\hline P35228 & NOS2 & Nitric Oxide Synthase 2 & 11 & 0.4695 & 0.0048 \\
\hline P10721 & KIT & $\begin{array}{l}\text { KIT Proto-Oncogene, Receptor Tyrosine } \\
\text { Kinase }\end{array}$ & 15 & 0.4926 & 0.0031 \\
\hline
\end{tabular}

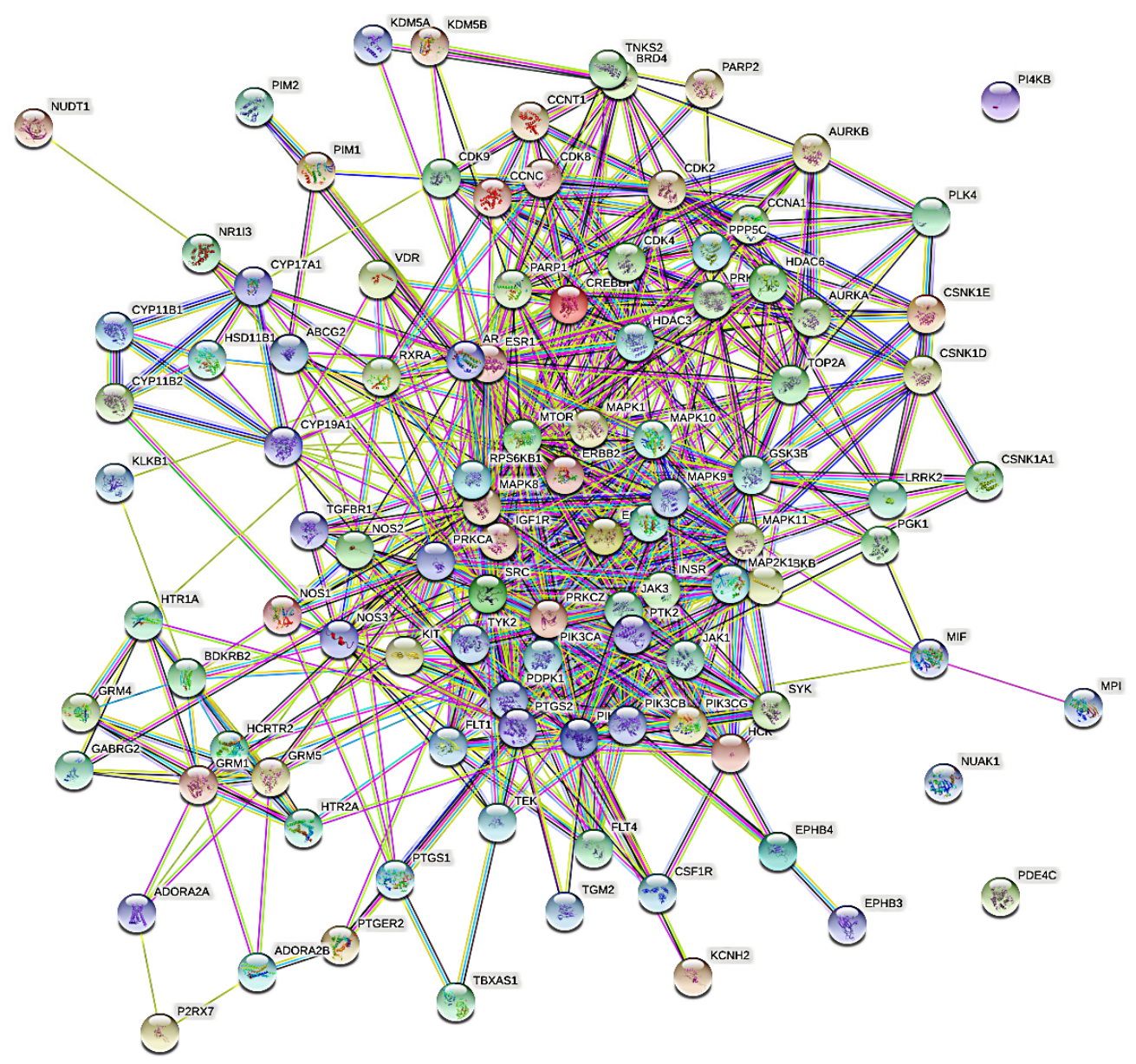

Fig. 3. The protein interaction network of drug-disease intersection target 


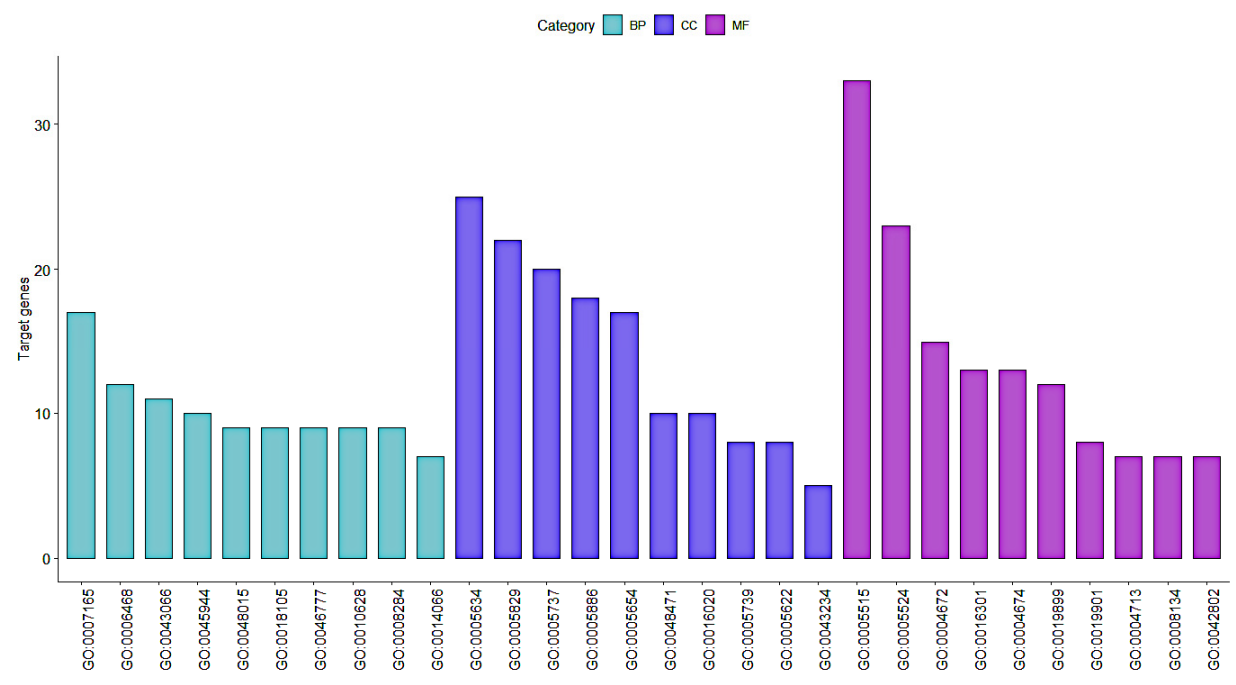

Fig. 4. Top 10 biological process enrichment results

\subsection{KEGG Pathway enrichment analysis}

KEGG pathway analysis was performed on core targets, and 18 important pathways were presented, the results are shown in Figure 5. The main signaling pathways include Pathways in cancer, PI3K-Akt signaling pathway, Proteoglycans in cancer, ErbB signaling pathway, HIF-1 signaling pathway related pathways.

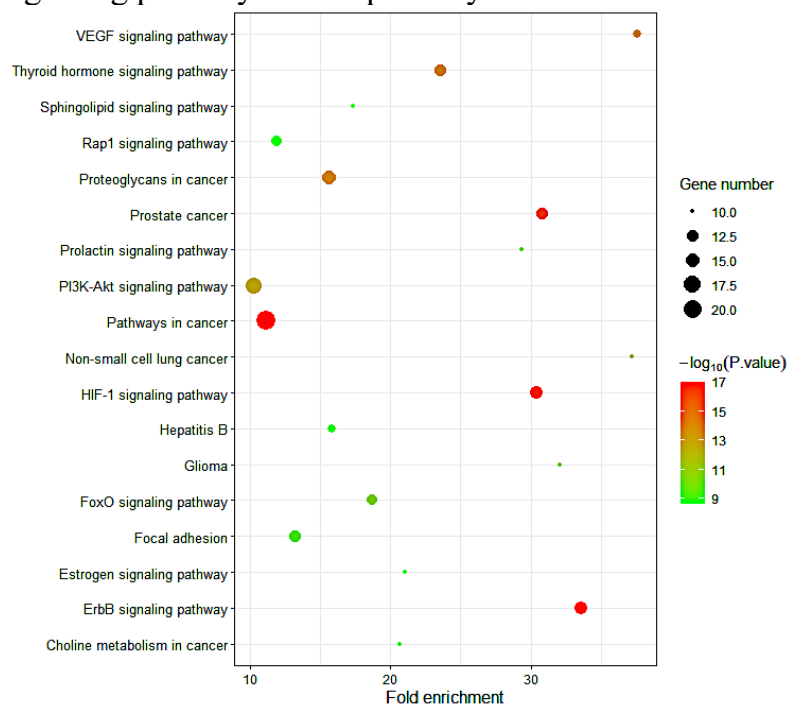

Fig. 5. The 18 pathways enriched by major hubs

\subsection{Pathway annotation diagram of OG's anti- BC effect}

We enter the core target into the KEGG database, KEGG mapping function will map the target into each pathway and mark the number of targets. 21 target proteins are involved in the Pathways in cancer.

\section{Discussion}

The analysis results of network pharmacology and bioinformatics show that SRC, PIK3CA, EGFR, MTOR and ESR1 may be the core targets of OG to inhibit BC. Src is an important anti-tumor drug target, and it is expressed at a high level in various tumors such as lung cancer, breast cancer, rectal cancer and pancreatic cancer [12]. Abnormally activated $\mathrm{Src}$ can be involved in the occurrence and development of tumors, such as apoptosis, proliferation, cell adhesion, migration , invasion, blood vessel formation and metastasis[13]. PIK3CA is a proto-oncogene, and mutations in the PIK3CA gene are associated with breast cancer hormone receptor expression and tumor progression[14].

GO biological process enrichment analysis reveals that the main biological process of OG's anti-BC effect may include signal transduction, protein phosphorylation, negative regulation of apoptotic process, positive regulation of transcription from RNA polymerase II promoter.

KEGG pathway enrichment analysis shows that OG anti-BC effects through regulating Pathways in cancer, PI3K-Akt signaling pathway, Proteoglycans in cancer, ErbB signaling pathway, HIF-1 signaling pathway related pathways. The occurrence of $\mathrm{BC}$ is related to a variety of factors, Studies have suggested that PI3K/AKT signaling pathway gene mutations often occur in $\mathrm{BC}[15]$.

\section{Conclusions}

A total of 104 potential targets were obtained, 33 of which are core targets for OG inhibit BC. GO biological process enrichment analysis and KEGG pathway enrichment analysis revealed 10 biological processes, cell composition, molecular functions and 18 pathways are closely related to the occurrence and development of inhibiting $\mathrm{BC}$, mainly involving Pathways in cancer, PI3K-Akt signaling pathway, Proteoglycans in cancer, ErbB signaling pathway, HIF-1 signaling pathway. In this study, we used the methods of network pharmacology and bioinformatics to preliminarily predict the molecular mechanism of $\mathrm{OG}$ inhibiting $\mathrm{BC}$, and provide a reference for the in-depth development of OG anti-tumor. 


\section{Acknowledgments}

The authors would like to thank the financial support from the Scientific Research Foundation of Hunan Provincial Education Department (20B415), the Technology Project Foundation of Tongren ([2018]48), the Technology Project Foundation of Tongren ([2019]55).

\section{References}

1. M. Ghoncheh, Z. Pournamdar, H. Salehiniya, Asian Pac J Cancer Prev, 17(S3), 43-46 (2016).

2. C. E. DeSantis, J. M. Ma, S. A. Goding, CA Cancer J Clin, 67(6), 439-448 (2017).

3. C. S. Benson, S. D. Babu, S. Radhakrishna, Dis Markers, 34(6), 395-405 (2013).

4. P. Xglv, R. Blt,M. A. Joore, Breast Cancer Res Treat, 165, 485-498 (2017).

5. P. Fox, A. Darley, E. Furlong, Eur J Oncol Nurs, 26, 63-82 (2017).

6. Chinese Society of Clinical Oncology (CSCO) Breast Cancer Diagnosis and Treatment Guidelines, People's Medical Publishing House, 24 (2017).

7. X. L. Zhang, Review on research progress of chemical constituents of Corydalis yanhusuo W. T. Wang[D], Shenyang, Shenyang Pharmaceutical University (2008).

8. N. G. Lubomira, Adelina,S. Anna, Metodieva, S. Angel, Galabov, Antiviral Research, 90(2) , A61, (2011).

9. N. Ivanovska, M. Hristova, S. Philipov, Pharmacol Res, 41(1):, 99-105, (2000).

10. M. Remichkova, P. Dimitrova, S. Philipov, N. Ivanovska, Fitoterapia, 80(7), 411-4 (2009).

11. N. Ivanovska, S. Philipov, Methods Find Exp Clin Pharmacol, 19(9), 79-83 (1997).

12. Q. Wang, L. Liu, Y. Le, L. J. Yan, Chin J Med Chem, 31(04), 312-319 (2021).

13. D. L. Wheeler, M. Iida, E. F. Dunn, Oncologist, 14(7):, 67-78 (2009).

14. Y. M. Deng, Y. J. Xu, Int J Lab Med, 37(15), 2110$2111+2114$ (2016).

15. I. A. Mayer, C. L. Arteaga, J Clin Oncol, 32(27), 2932-4 (2014). 